\title{
Studies of RF sheaths and diagnostics on IShTAR
}

K. Crombé', S. Devaux, R. D'Inca, E. Faudot, H. Faugel, H. Fünfgelder, S. Heuraux, J. Jacquot, F. Louche, J. Moritz, R. Ochoukov, M. Tripsky, D. Van Eester, T. Wauters, and J.-M. Noterdaeme

Citation: AIP Conference Proceedings 1689, 030006 (2015); doi: 10.1063/1.4936471

View online: http://dx.doi.org/10.1063/1.4936471

View Table of Contents: http://aip.scitation.org/toc/apc/1689/1

Published by the American Institute of Physics

\section{Articles you may be interested in}

First experimental results on the IShTAR testbed

AIP Conference Proceedings 1689, 050010050010 (2015); 10.1063/1.4936498

Designing the IShTAR antenna: Physics and engineering aspects

AIP Conference Proceedings 1689, 070016070016 (2015); 10.1063/1.4936523

Full wave propagation modelling in view to integrated ICRH wave coupling/RF sheaths modelling AIP Conference Proceedings 1689, 050008050008 (2015); 10.1063/1.4936496 


\title{
Studies of RF sheaths and diagnostics on IShTAR
}

\author{
K. Crombé ${ }^{1,2, a)}$, S. Devaux ${ }^{3}$, R. D’Inca ${ }^{4}$, E. Faudot ${ }^{3}$, H. Faugel $^{4}$, H. Fünfgelder ${ }^{4}$, S. \\ Heuraux $^{3}$, J. Jacquot ${ }^{4}$, F. Louche ${ }^{2}$, J. Moritz ${ }^{3}$, R. Ochoukov ${ }^{4}$, M. Tripsky ${ }^{2}$, D. Van \\ Eester $^{2}$, T. Wauters ${ }^{2}$ and J.-M. Noterdaeme ${ }^{1,4}$ \\ ${ }^{1}$ Department of Applied Physics, Ghent University, Ghent, Belgium \\ ${ }^{2}$ LPP-ERM/KMS, Royal Military Academy, Brussels, Belgium \\ ${ }^{3}$ YIJL, UMR7198 CNRS-Université de Lorraine, Nancy, France \\ ${ }^{4}$ Max-Planck-Institut für Plasmaphysik, Garching, Germany \\ ${ }^{a}$ Corresponding author: Kristel.Crombe@UGent.be
}

\begin{abstract}
IShTAR (Ion cyclotron Sheath Test ARrangement) is a linear magnetised plasma test facility for RF sheaths studies at the Max-Planck-Institut für Plasmaphysik in Garching. In contrast to a tokamak, a test stand provides more liberty to impose the parameters and gives better access for the instrumentation and antennas. The project will support the development of diagnostic methods for characterising RF sheaths and validate and improve theoretical predictions. The cylindrical vacuum vessel has a diameter of $1 \mathrm{~m}$ and is $1.1 \mathrm{~m}$ long. The plasma is created by an external cylindrical plasma source equipped with a helical antenna that has been designed to excite the $m=1$ helicon mode. In inductive mode, plasma densities and electron temperatures have been characterised with a planar Langmuir probe as a function of gas pressure and input RF power. A 2D array of RF compensated Langmuir probes and a spectrometer are planned. A single strap RF antenna has been designed; the plasma-facing surface is aligned to the cylindrical plasma to ease the modelling. The probes will allow direct measurements of plasma density profiles in front of the RF antenna, and thus a detailed study of the density modifications induced by RF sheaths, which influences the coupling. The RF antenna frequency has been chosen to study different plasma wave interactions: the accessible plasma density range includes an evanescent and propagative behaviour of slow or fast waves, and allows the study of the effect of the lower hybrid resonance layer.
\end{abstract}

\section{Introduction}

The IShTAR (Ion cyclotron Sheath Test ARrangement) project, at the Max-Planck-Institut für Plasmaphysik in Garching (Germany), aims at studying antenna near-fields and RF sheath effects in the presence of a plasma and magnetic field in the framework of a European collaboration. Consequences of plasma wave interactions - as for example a modification of the plasma density in front of the antenna, the creation of static electric fields, material sputtering and the formation of hot spots - are observed on different experimental devices and are detrimental and potentially dangerous for antenna operation. The measurements will help theoretical predictions on RF sheaths and will guide theoretical modelling. IShTAR operates at tokamak edge-like conditions for density $\left(\mathrm{n}_{\mathrm{e}}\right)$ and temperature. IShTAR has been equipped with a helicon plasma source. The characterisation of the plasma is ongoing. A single strap ICRF antenna is under construction; the design was simplified as far as possible to facilitate the sheath modelling activities. In addition, IShTAR is intended to serve as a test environment for the development of antenna and sheath diagnostics.

\section{The setup and plasma characteristics}

\section{Technical specifications}

A detailed description of the capabilities of the test bed and the first experiments is given in [1]. A schematic overview of the different components is shown in fig. 1 (taken from [1]). The testbed consists of a main vessel and a separate chamber where the plasma is created by a helical antenna (view 2). The plasma source is connected to the main vessel by a port with a diameter of $0.4 \mathrm{~m}$. The dimensions of the port opening limit the plasma volume, as it can be seen on 
the view with the argon plasma in fig. 1. The bolts that can be observed on the upper left side approximately show the location where the first ICRF antenna will be installed. This antenna can be fed by transmission lines that are connected to the ASDEX generators, with ample amount of power available. For the first operations, however, a low power (up to $1 \mathrm{~kW}$ ) generator will be used, with a frequency range [0.1 - $100 \mathrm{MHz}]$. The plasma is created by a helical antenna (outside the glass tube on view 2). Four small magnetic coils surround the glass tube. At present the maximal current in the small coils is limited by the available power source to $1 \mathrm{kA}$, which corresponds to a $\mathrm{B}_{0}$-field of $0.1 \mathrm{~T}$. The small coils can obtain a maximal nominal current of $1.5 \mathrm{kA}$ and $4.0 \mathrm{kA}$ in pulsed mode. The helical antenna is 1 $\mathrm{m}$ long and has a diameter of $0.6 \mathrm{~m}$. For the given value of the magnetic field in the plasma source the minimum $\mathrm{n}_{\mathrm{e}}$ for a helicon wave to propagate is estimated to be $\approx 710^{16} \mathrm{~m}^{-3}$. This still needs to be verified and the helicon operational mode has not yet been obtained. The cylindrical main vessel has a length of $1.1 \mathrm{~m}$ and a $1 \mathrm{~m}$ diameter, different ports are available for installing diagnostics. The main magnetic field is created by two big coils around the main vessel. A value of $0.1 \mathrm{~T}$ is obtained for a current of $2.4 \mathrm{kA}$ in the big coils. The maximal current in the big coils is $8 \mathrm{kA}$ (for 10 s), which corresponds to a magnetic field of $0.24 \mathrm{~T}$. The vacuum system is connected on the back flange and consists of a pre-vacuum pump to reach a pressure of $10^{-2} \mathrm{mbar}$, and a turbomolecular pump creates high vacuum $\left(10^{-6} \mathrm{mbar}\right.$ level). A typical discharge lasts $5-10 \mathrm{~s}$ and the shots have a high repetition rate (10 - 20 pulses per hour are possible).

\section{Characterisation of the plasma}

For a first characterisation of the plasma one Langmuir probe has been installed at the back flange (indicated as component \#17 in fig. 1). The probe design had not been optimised for the experiment; an available probe was used to be able to initiate measurements. A set of experiments has been performed [2] with varying gas pressure, between 0.2 and $2.010^{-3} \mathrm{mbar}$, and varying input power for the helical antenna, between $300 \mathrm{~W}$ and $800 \mathrm{~W}$. Density and temperature values have been derived, and are shown in the figures. In fig. 2 (a) and (b) the density and temperature are plotted as a function of the gas pressure, at a fixed input power of $600 \mathrm{~W}$ at the operating RF frequency $f=11.76$ $\mathrm{MHz}$, and in (c) and (d) at the gas pressure was kept constant at values of 0.2 and $2.010^{-3} \mathrm{mbar}$, while the coupled power was varied. The accessible plasma parameters, in the present configuration, are of the order $10^{15}-10^{16} \mathrm{~m}^{-3}$ for the density and $5-10 \mathrm{eV}$ for the temperature. The density is - although already at the level of typical antenna and plasma edge values in a tokamak - rather low for the coupled power. This is possibly due to the location of the probe, which is not placed in the centre, but at the edge of the cylindrical plasma column. In fig. 2 can also be seen that the temperature increases by a mere $20 \%$ when the density is increased by a factor of three. This might indicate that the absorbed power influences the Langmuir probe measurements, or that a big part of the energy is lost somewhere; more test should be done to give a clear answer. The sensitivity and accuracy of the actual Langmuir probe measurements are not sufficient to be able to optimise the performance of the plasma source. Improvements to the Langmuir probe system are foreseen (RF compensated probes, and a better accuracy in the bias sampling). The maximal power that is presently available for the helical antenna is $3 \mathrm{~kW}$. At that power level the helicon mode of operation, which is expected to lead to a significant increase in density, was not yet reached; an upgrade is planned.

\section{Characteristics of the RF antenna}

\section{Design of the antenna}

Different design options for the first antenna have been made [3]. Since IShTAR is meant to contribute to the studies of RF sheath effects, a single strap antenna with a simple geometry will be installed at first, such that the measurements can be compared directly with numerical simulations. The experiments will concentrate on the dependence of the 2D potential pattern and antenna near-field characteristics, on the plasma parameters (density, temperature) and power to the antenna. Later on, a rotatable antenna will be considered to study variations of the magnetic field angle. Gradually more complicated antennas - with multiple straps, allowing to look into the impact of phasing - will be developed and tested. Differences in phasing will change the electric fields generated by the antenna and thus the potential pattern and the sheath properties, increasing the challenge for the numerical models.

For the first single strap antenna the modelling was done with two commercial software packages: CST-Micro Wave Studio [4] and COMSOL [5], and for vacuum, dielectric and plasma conditions. For vacuum and dielectric with modest relative permittivity $\left(\epsilon_{r}\right.$ of the order of $100 \mathrm{~F} / \mathrm{m}$ ) both codes show good agreement. For dielectric media with high $\epsilon_{r}$ and for plasma parameters both codes have difficulties to find a converged solution, due to numerical limits $[6,7]$. Since the plasma is limited in volume by the connecting flange between the plasma source and the main vessel, an antenna with curved strap, which follows the cylindrical plasma shape, was chosen as the best solution. In that way, the complete strap is immersed in the plasma, and located at a fixed value for the magnetic field, the plasma density 


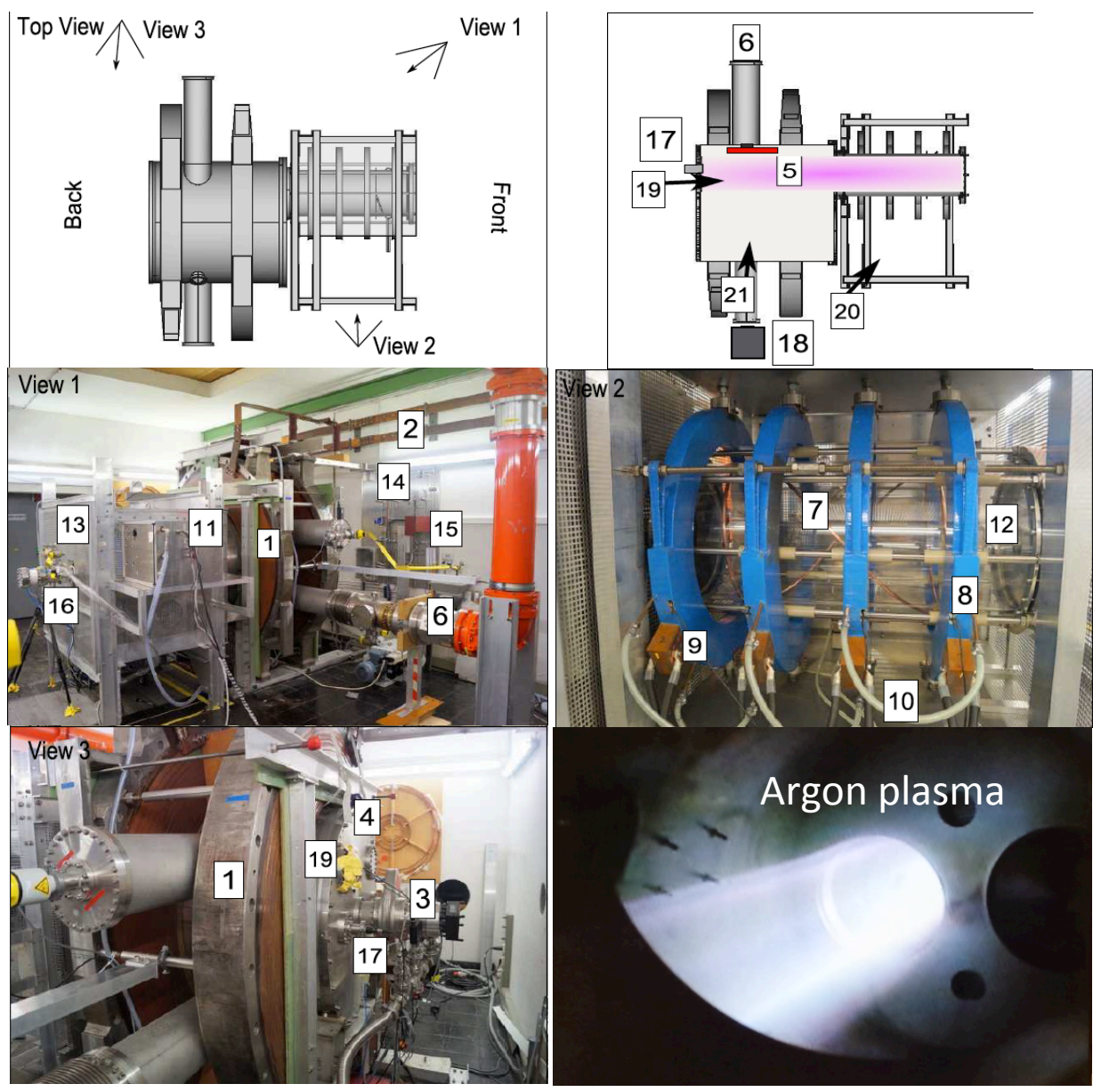

Main vessel

1. Big coils

2. Coils power lines

3. Vacuum pump

4. Back flange ICRF System

5. ICRF antenna

6. Transmission

Plasma source

7. Helicon antenna

8. Small coils

9. small coils power

10. Cooling system

11. Matching system

12. Glass tube

13. RF Radiation shield

Gas feeding

14. Manifold panel

15. Main valve

16. Gas inlet

Instrumentation

17. Langmuir probe

18. Spectrometer

19. 20. 21. Cameras

FIGURE 1. Overview of the IShTAR set-up with the different components. On the bottom right picture an argon plasma inside the main vessel is shown.

and temperature (fig. 3). Although it is more difficult to construct, it has the advantage that it potentially simplifies the modelling efforts, because of the symmetry. This is an important aspect of the test facility compared to an existing fusion machine. The design and associated numerical simulations are finished and the construction is starting. The present planning is that the construction, installation and first tests of the antenna in IShTAR are done in 2015.

\section{Choice of the frequency}

A parametric study was done to chose the most "sheath-relevant" frequency for the antenna [8], based on the density ranges that have been measured with the Langmuir probe.

Typical tokamak parameters The cold plasma description is adequate when doing wave studies in the edge of a tokamak, where temperatures are sufficiently low (a few tens of eV) [9]. Antenna design is routinely done assuming the density is low enough to neglect the presence of the plasma at the antenna straps inside the antenna box. Densities vary from nearly zero at the antenna launcher to $10^{17}-10^{18} \mathrm{~m}^{-3}$ at the plasma edge. Behind the last closed flux surface the density typically decays exponentially with a decay length of a few $\mathrm{cm}$. For typical tokamak edge conditions the lower hybrid resonance layer, the low density fast wave (FW) cut-off and the slow wave (SW) cut-off are crossed going from the antenna strap towards the central plasma. Depending on the exact parameters, the cut-offs and resonance occur inside the antenna box or in front of it. Just because in antenna codes the low density region is mostly not taken into account and replaced by a vacuum layer, the reality that the plasma impacts sensitively on the wave behaviour is typically sidestepped, which does not allow to adequately describe the near-field pattern that is crucial for describing 

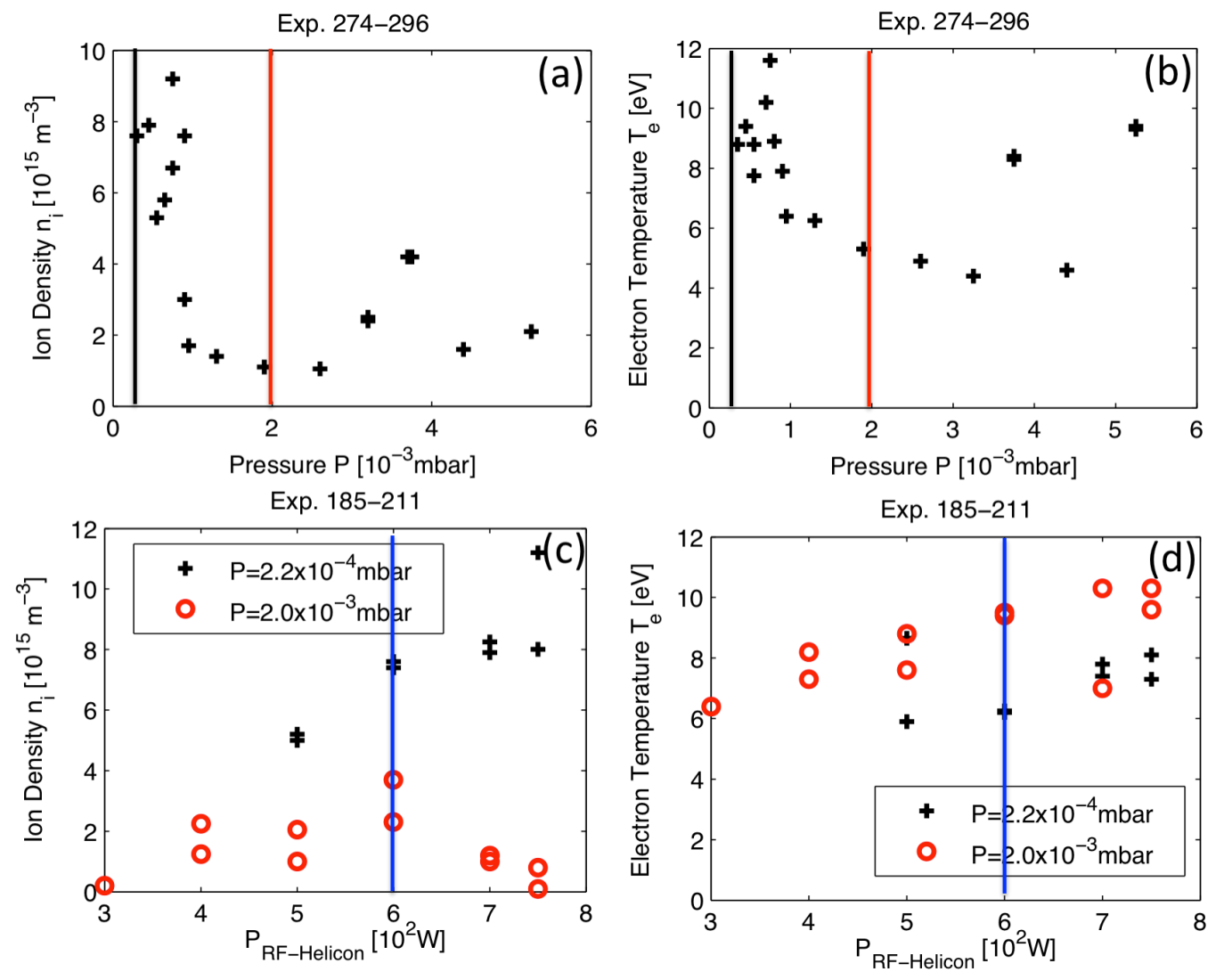

FIGURE 2. (a) and (b) show the density and temperature as they have been measured by the Langmuir probe as a function of the gas pressure at a constant coupled power of $600 \mathrm{~W}$ (vertical line in blue on the bottom figures). (c) and (d) are scans in coupled power, at two different constant gas pressures (vertical lines in black and red on the top figures).

the non-resonant acceleration of particles, thought to be the origin of sputtering. At the lower hybrid resonance one of the roots of the dispersion equation (i.e. the SW in the decoupled formulation) changes its behaviour from propagative (for densities just below the LHR) to evanescent (for densities above the LHR). In fig. 4(a) the perpendicular wave vector $k_{\perp}^{2}$ is plotted as a function of the density in the range $\left[10^{13}-10^{20} \mathrm{~m}^{-3}\right]$. In the low density region, both roots approach the vacuum limit, $k_{\perp}^{2}=k_{0}^{2}-k_{\mid l}^{2}$, as expected, while at higher densities representative for the core one roots is propagative $(\mathrm{FW})$ and the other is deeply evanescent $(\mathrm{SW})$. The calculations were done for parameters relevant for the edge region of tokamak plasmas and for radio frequency heating: a magnetic field $\mathrm{B}_{0}=2.0 \mathrm{~T}$, an antenna frequency $f$ $=50 \mathrm{MHz}$, the dominant parallel wave component $k_{\|}=5 \mathrm{~m}^{-1}$ of a typical dipole spectrum, a temperature of $10 \mathrm{eV}$, and for a plasma composed of $95 \%$ of deuterium and $5 \%$ of hydrogen. Under these conditions the LHR (i.e. where $k_{\perp}^{2}$ goes to infinity) is located just below $10^{17} \mathrm{~m}^{-3}$ as can be seen in the figure. The cut-off frequencies (i.e. where $k_{\perp}^{2}$ goes through zero) for slow and fast wave are also visible on the plot. The cut-offs are located around density values of the order of $510^{13} \mathrm{~m}^{-3}$ and $10^{18} \mathrm{~m}^{-3}$ respectively.

IShTAR parameters To be useful, the best solution is to mimic as close as possible the tokamak conditions. However, the magnetic field in the test stand is significantly lower than in a tokamak: maximally $\mathrm{B}_{0}=0.1 \mathrm{~T}$. In fig. 4(b) $k_{\perp}^{2}$ is plotted for $\mathrm{B}_{0}=0.1 \mathrm{~T}$ and a $100 \%$ argon plasma. Argon is the gas used for the initial operation phase of IShTAR. Operation with other gases, such as helium and hydrogen, are foreseen for future campaigns, but they are found to not significantly alter the choice of the operating window. The frequency had to be adapted to $15 \mathrm{MHz}$ to obtain a tokamak-like behaviour. In fig. 4(c) the result is shown for $\mathrm{B}_{0}=0.24 \mathrm{~T}$, which is the maximal field that can be created by the big coils of IShTAR, and an operating frequency of $30 \mathrm{MHz}$ (lowest frequency possible for the ASDEX generators). Under these conditions a density around $10^{17} \mathrm{~m}^{-3}$ is most relevant to study $\mathrm{SW}$ behaviour. An important 

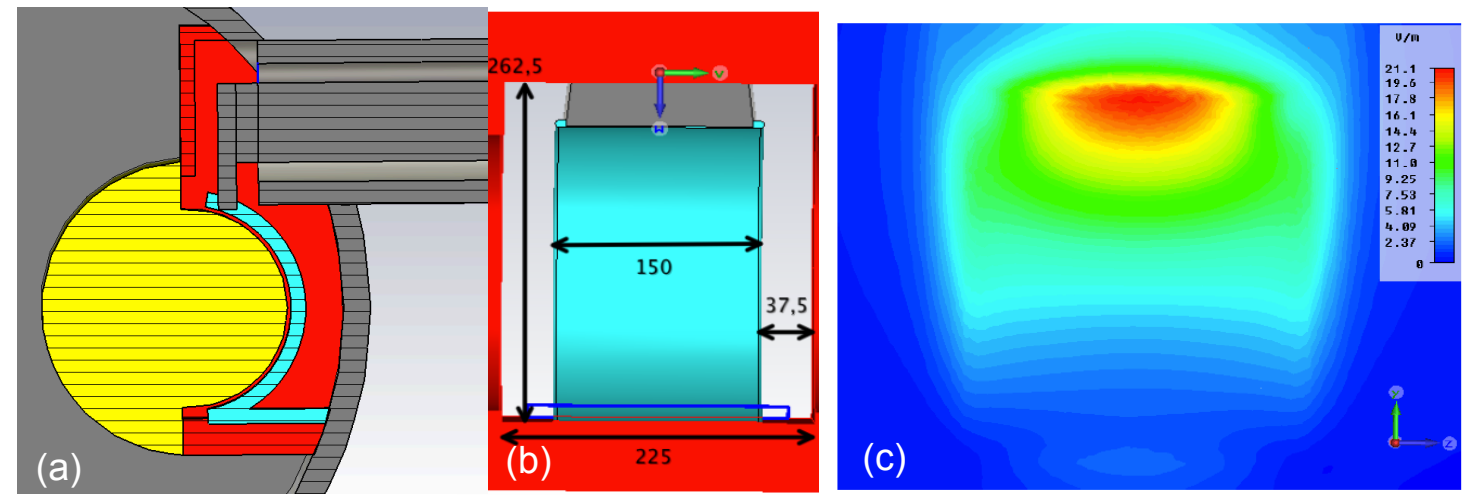

FIGURE 3. Final design of the single strap ICRF to be built into IShTAR, (a) side view with the size of the plasma in yellow, (b) frontal view (dimensions in $\mathrm{mm}$ ) and (c) the vacuum $E$-field amplitude at $1 \mathrm{~cm}$ away (in radial direction) from the edge of the antenna box

aspect of the sheath studies is the impact of the interaction of both wave types. The SW is propagative at densities between $S=0$ and $k_{0}^{2} S=k_{/ /}^{2}$ and carries energy away from the antenna to the LHR (in [10] and [11] examples are given of the SW exponentially decaying or adding a short wavelength propagation signature to the electric field structure close to the launcher). Such behaviour is at odds with the usual assumption that a FW antenna merely excites FWs which need to tunnel through the evanescence layer to reach the $k_{0}^{2} R=k_{/ /}^{2}$ cut-off and that the SW plays no role in the process. Understanding the interaction of the two modes seems crucial and should experimentally be addressed in IShTAR. The choice of the frequency for the antenna operation is important. In fig. 5 four different operating frequencies are plotted in the range [15-60 MHz]. For low frequencies (e.g. the $15 \mathrm{MHz}$ case) the wave behaviour is similar to the standard tokamak scenario, with a slow and fast wave cut-off at clearly distinct densities and a typical LHR that only affects the SW, since the FW is still evanescent for that density. However for increasing frequencies (e.g. the $30 \mathrm{MHz}$ case), the SW and FW cut-offs move closer to each other, and the FW becomes propagative at densities below the LHR (e.g. the $45 \mathrm{MHz}$ case). At that moment (and this continues for even higher frequencies, e.g. the $60 \mathrm{MHz}$ case) the effect of the resonance is visible on both waves as a sudden change from propagative to evanescent behaviour. The full solutions of the 4th order dispersion relation are clearly different from the decoupled FW and SW solutions. Therefore, for studying the interaction characteristics of the RF waves and the plasma in the region between the antenna and the LCFS in a tokamak, frequencies of $\sim 10 \mathrm{MHz}$ are envisaged. In addition at this frequency (which is considerably lower than for a tokamak antenna) the LHR is pushed towards lower densities - level $10^{16} \mathrm{~m}^{-3}$ for $15 \mathrm{MHz}$ instead of $10^{18} \mathrm{~m}^{-3}$ for the $50 \mathrm{MHz}$ example - which are easier to reach in the test bed.

\section{Ionisation of the gas by the ICRF antenna}

The effect of the ICRF antenna on the plasma ionisation was investigated using the code Tomator [12], setting a particle confinement time determined by the ion sound velocity and the length of the test chamber. The plasma density created by the antenna, depends moreover on the RF power coupled to the plasma. Figure 6 illustrates some cases for a helium and a hydrogen plasma. The estimated ionisation densities are relatively similar for the two species. For a power of $1 \mathrm{~kW}$ in helium for example (expected as the initial operating condition), the density created by the antenna will be $2.210^{15} \mathrm{~m}^{-3}$, which is of the same order of magnitude as the density that is presently obtained by the helical plasma source. Therefore it will be difficult to distinguish the sheath effects from the pure ionisation effects. The ionisation will be less for a higher background plasma density, but therefore a more powerful generator needs to be installed on the helical antenna.

\section{Enlarging the diagnostic capabilities}

To ensure that the primary goal - experimentally characterising the wave-plasma interaction close to RF launchers can be reached, IShTAR needs proper diagnostics. Planned are (i) an array of probes, (ii) an IR camera, and (iii) a spectroscopic system for E-field measurements. To allow for an automatic collection of all data during the experiments, an integrated data acquisition system is being developed. Other diagnostics, such as a mass spectroscopy system to get 

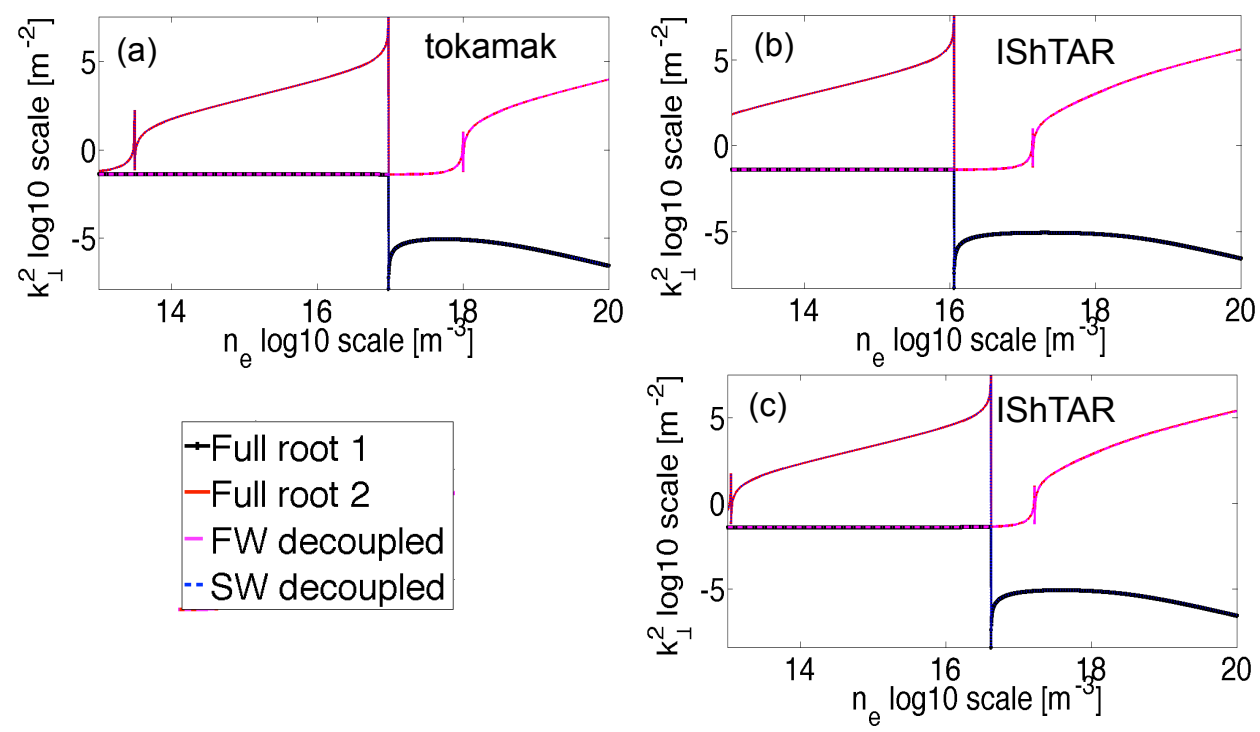

FIGURE 4. (a) $k_{\perp}^{2}$ values for full and decoupled solutions as a function of the plasma density for typical tokamak parameters: $\mathrm{B}_{0}=$ $2.0 \mathrm{~T}$, freq $=50 \mathrm{MHz}$. (b) $k_{\perp}^{2}$ for standard IShTAR parameters: magnetic field $\mathrm{B}_{0}=0.1 \mathrm{~T}$, freq $=15 \mathrm{MHz}$ and a pure argon plasma (with partial ionisation $\mathrm{Ar}^{8+}$ ). (c) $k_{\perp}^{2}$ for $\mathrm{B}_{0}=0.24 \mathrm{~T}$ and freq $=30 \mathrm{MHz}$.

information on the plasma composition, and a LIF (Laser Induced Fluorescence) diagnostic based on a laser diode to determine the ion distribution function, can be added later.

- (i) The experiments with the fixed Langmuir probe have shown that an upgrade of the system is required [2]. RF compensated probes will be needed as well as a better accuracy in the bias sampling. The design of a 2D probe array is ongoing in order to have a more complete picture of the density profiles, and of the density modifications due to the electromagnetic fields. First measurements have been taken with a probe that was mounted on a movable arm. The arm was plunged into the plasma from the edge towards the centre in consecutive discharges.

- (ii) An IR camera is needed to improve the performance of the plasma source. It is essential to understand where the power is deposited, also to avoid overheating and damage to the glass tube. Next to that, the IR camera can be used in the main vessel and provide information on the formation of hot spots on the antenna structure.

- (iii) A spectrometer was used to look at the line intensity of argon lines. Of particular interest and being looked into at this moment, is the possibility to directly measure the RF electric field magnitude. The options considered are detection by Stark effect, satellites of He I lines caused by forbidden transitions in a strong electric field environment, or the detection of Lyman- $\alpha$ light from a neutral hydrogen beam in the metastable $2 s$ - state.

\section{Predictions for the density depletion due to RF fields in IShTAR}

The theory and modelling of RF sheath physics in a tokamak environment has been advanced in recent years $[10,11$, $13,14,15]$. The physics that describes the interplay between RF waves and the plasma density close to conducting surfaces is complex; at least two sets of non-linear equations (for ions and electrons) are needed, and they act on very different time scales: the slow (transport processes) and fast (RF frequency) time scales differ by several orders of magnitude. In [16] an expression is derived for the density modifications of electrons and ions, i.e. on the slow timescale, but taking the effect of DC RF fields into account. The model includes the parallel flow and the usual electrostatic potential as well as the RF ponderomotive force. The above demonstrates that the parallel energy and the electrostatic and ponderomotive potential play a similar role in modifying the density. As all these quantities are correlated, solely looking at the above is somewhat misleading. So far only 1-D density variations were looked into. In a next step a more complete 2-D description will be developed, which also includes the creation of RF induced convective cells. In case the magnetic field is strong the perpendicular drift contributions can be neglected in the 


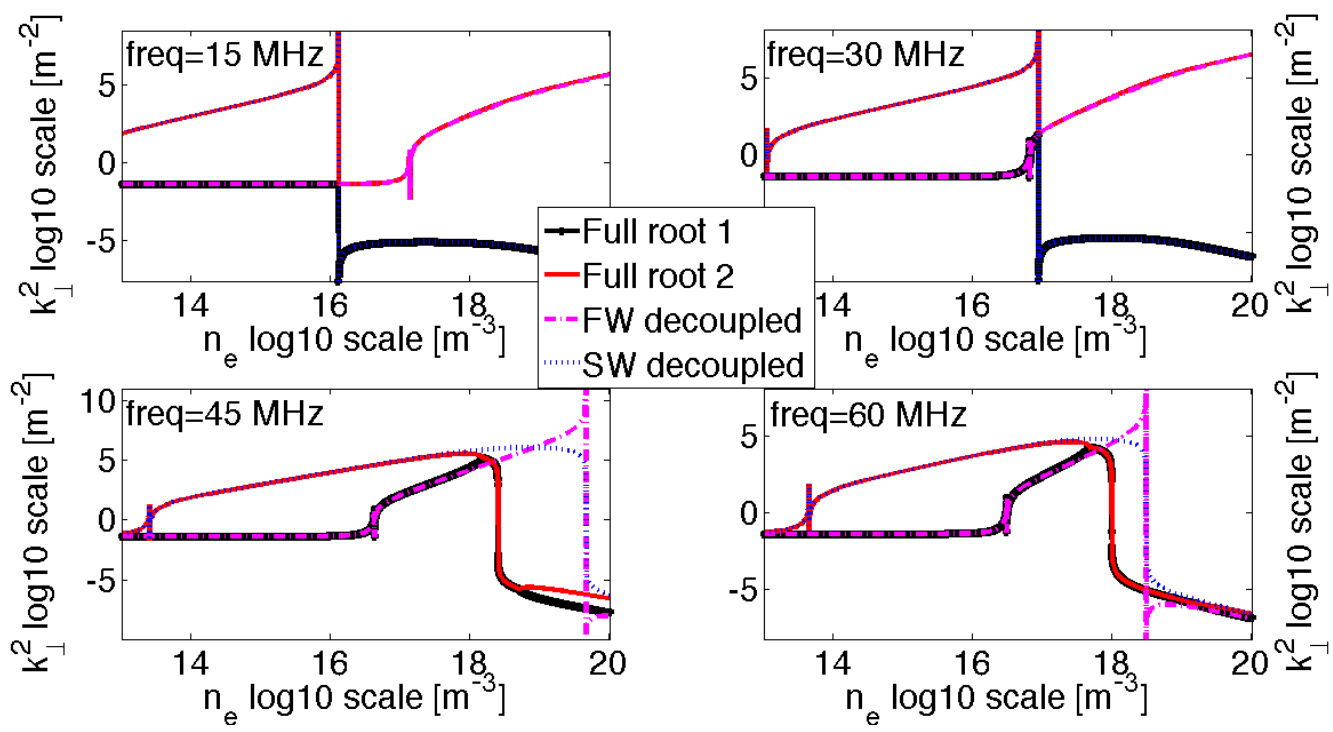

FIGURE 5. $k_{\perp}^{2}$ values for full and decoupled solutions as a function of the plasma density for similar parameters as in fig. $4(\mathrm{~b})$, but different frequencies: $15 \mathrm{MHz}, 30 \mathrm{MHz}, 45 \mathrm{MHz}$ and $60 \mathrm{MHz}$.

\begin{tabular}{|c|c|c|c|c|c|c|c|c|c|}
\hline HELIUM & & & & & HYDROGEN & & & & \\
\hline RFcoupled & pHe [mbar] & Bt & ne $[\mathrm{m}-3]$ & Te $[\mathrm{eV}]$ & RFcoupled & pH2 [mbar] & Bt & ne $[\mathrm{m}-3]$ & Te $[\mathrm{eV}]$ \\
\hline $500 \mathrm{~kW}$ & $1.0 \mathrm{e}-5$ & 0.1 & 0 & -- & $500 \mathrm{~kW}$ & $1.0 \mathrm{e}-5$ & 0.1 & 0 & -- \\
\hline $1 \mathrm{~kW}$ & $1.0 \mathrm{e}-4$ & 0.1 & $1.4 \mathrm{e} 15$ & 22 & $5 \mathrm{~kW}$ & $1.0 \mathrm{e}-4$ & 0.1 & $6.6 \mathrm{e} 15$ & 17 \\
\hline $5 \mathrm{~kW}$ & $1.0 \mathrm{e}-4$ & 0.1 & $6.8 \mathrm{e} 15$ & 22 & $20 \mathrm{~kW}$ & $1.0 \mathrm{e}-4$ & 0.1 & $3.2 \mathrm{e} 16$ & 15 \\
\hline $20 \mathrm{~kW}$ & $1.0 \mathrm{e}-4$ & 0.1 & $2.6 \mathrm{e} 16$ & 23 & $100 \mathrm{~kW}$ & $1.0 \mathrm{e}-4$ & 0.1 & $1.2 \mathrm{e} 17$ & 16 \\
\hline $1 \mathrm{~kW}$ & $1.0 \mathrm{e}-3$ & 0.1 & $2.2 \mathrm{e} 15$ & 9.3 & $100 \mathrm{~kW}$ & $1.0 \mathrm{e}-3$ & 0.1 & $2.8 \mathrm{e} 17$ & 4.1 \\
\hline $10 \mathrm{~kW}$ & $1.0 \mathrm{e}-3$ & 0.1 & $2.1 \mathrm{e} 16$ & 9.4 & $500 \mathrm{~kW}$ & $1.0 \mathrm{e}-3$ & 0.1 & $8.8 \mathrm{e} 17$ & 4.5 \\
\hline
\end{tabular}

FIGURE 6. Estimate of the plasma created by the antenna for a certain amount of RF power coupled to the plasma, runs performed by the Tomator $0 \mathrm{D}$ code [12].

equation characterising the parallel flow. The expression for the density $\left(N_{0}\right)$ variation is then given by:

$$
N_{0}=N_{0, \text { ref }} \exp \left(-\frac{1}{v_{t}^{2}}\left(\frac{v_{\|}^{2}}{2}+\frac{q \Phi}{m}+\Theta\right)\right)
$$

where $N_{0, \text { ref }}$ is the reference density, $v_{t}$ is the thermal and $v_{\|}$the parallel velocity, $q$ and $m$ are resp. charge and mass of the species, $\Phi$ is the usual electrostatic potential and $\Theta$ is the potential associated with the RF ponderomotive force. The expected density variations from a fixed reference value towards a metallic wall are illustrated in fig. 7 for different strengths of electric fields. The reference density was chosen to be $10^{16} \mathrm{~m}^{-3}$, obtainable in IShTAR, it is imposed at the right hand side of the simulation domain (see fig. 7). The antenna is modelled as a layer of constant current. The simulation was done for a pure deuterium plasma, a frequency of $10 \mathrm{MHz}, \mathrm{B}_{0}=0.1 \mathrm{~T}$ and $v_{\|}=1.1 v_{t}$. The model can directly be tested on IShTAR, be it that 2-D effects first should be added. The array of probes will provide local measurements of the density close to the antenna, whereas the spectroscopic system should allow to get an estimate of the electric fields.

\section{Conclusions}

It was found that the accessible plasma parameters in IShTAR, in the present configuration, are of the order $10^{15}-10^{16}$ $\mathrm{m}^{-3}$ for the density and $5-10 \mathrm{eV}$ for the temperature. Improvements to the probe design for future measurements have been identified. An increase of the power coupled to the plasma should increase the density further, possibly exciting 


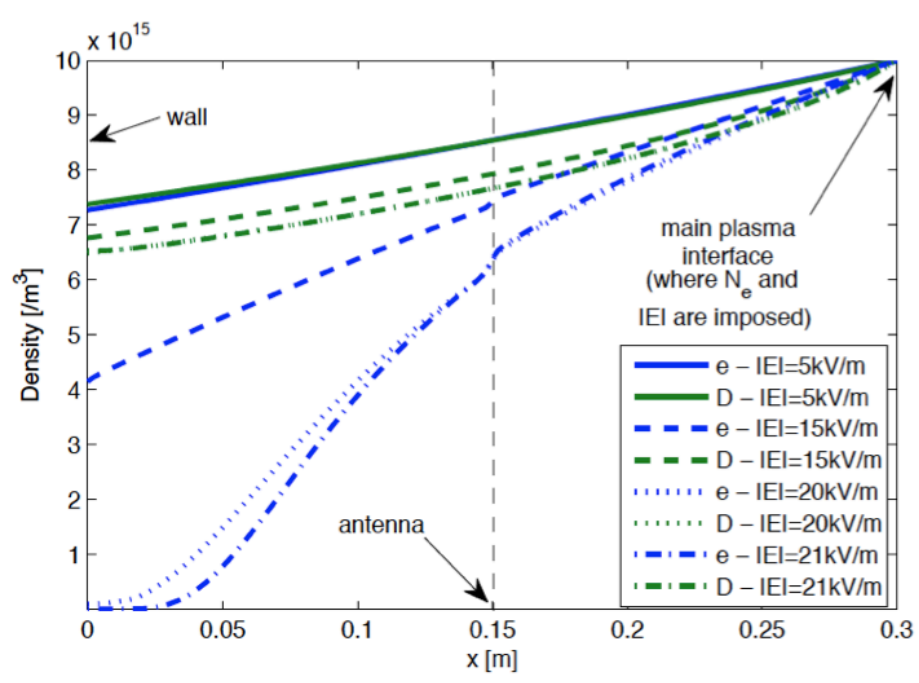

FIGURE 7. Estimated plasma density depletion for varying E-fields.

the helicon mode. A curved single strap antenna is under construction. The best operating frequency for IShTAR was identified to be around $10 \mathrm{MHz}$, based on the cold plasma dispersion relation of the waves. The diagnostic capabilities are expanding and will concentrate on direct verifications of sheath models.

\section{Acknowledgments}

The authors want to thank the technical staff at IPP-Garching and LPP-ERM/KMS, in particular F. Fischer, G. Siegl and M. Berte. The work received support from the Research Foundation Flanders (G0B3115N). This work has been carried out within the framework of the EUROfusion Consortium and has received funding from the Euratom research and training programme 2014-2018 under grant agreement No 633053. The views and opinions expressed herein do not necessarily reflect those of the European Commission. The views and opinions expressed herein do not necessarily reflect those of the European Commission.

\section{REFERENCES}

[1] R. D’Inca, K. Crombé et al., "IShTAR: a test facility to investigate sheath effects during Ion Cyclotron Resonance Heating", in preparation

[2] R. D'Inca, J. Jacquot et al., "First experimental results on the IShTAR testbed", this conference

[3] F. Louche, J. Jacquot et al., "Designing the IShTAR antenna: physics and engineering aspects", this conference

[4] CST STUDIO SUITE, CST AG, Germany, www.cst.com

[5] Comsol Multiphysics, www.comsol.com

[6] K. Crombé, J. Jacquot, F. Louche and D. Van Eester, "Numerical challenges in modelling near-antenna field behaviour in cold plasmas", Proceedings of the 41st EPS Conference on Plasma Physics, 23rd - 27th June 2014, Berlin, Germany, ECA, Vol. 38F (2014), P1.039

[7] L. Lu et al., "Wave coupling in the magnetized plasma edge: impact of a finite, inhomogeneous density inside the antenna box", this conference

[8] K. Crombé and D. Van Eester, "Coupled and decoupled solutions of the cold plasma dispersion relation", in preparation

[9] T.H. Stix. "Waves in Plasmas", AIP, New York (1992)

[10] D. Van Eester, K. Crombé and V. Kyrytsya, Plasma Phys. Control. Fusion 55 (2013) 025002

[11] D. Van Eester, K. Crombé and V. Kyrytsya, Plasma Phys. Control. Fusion 55 (2013) 055001

[12] T. Wauters et al, Plasma Physics and Controlled Fusion 53 (2011) 125003.

[13] L. Colas et al., Phys. Plasmas 19 (2012) 092505

[14] J. Jacquot et al., Phys. Plasmas 21 (2014) 061509

[15] A. Ngadjeu, E. Faudot, J. Gunn, L. Colas and S. Heuraux, AIP Conf. Proc. 1187, 161 (2009)

[16] D. Van Eester, K. Crombé and L. Lu, "Wave induced density modification in RF sheaths and close to wave launchers", this conference 